Daniel Moreau

Université de Sherbrooke (Canada)

François Larose

Université de Sherbrooke (Canada)

Jonathan Smith

Université de Sherbrooke (Canada)

\section{Lintention de persister d'étudiants québécois en éducation préscolaire et en enseignement primaire en contexte de pandémie}

\author{
Student intention to persist in preschool and elementary school education in
}

Québec in a pandemic context

doi: $10.18162 / f p .2020 .687$

\section{ésumé}

Cet article a pour objectif de documenter l'intention de persister dans leur formation et leur choix de carrière en enseignement des étudiants en éducation préscolaire et en enseignement primaire dans le contexte actuel de pandémie. Une analyse de contenu lexicométrique de données provenant d'entrevues réalisées auprès d'un échantillon de 33 étudiants a été réalisée. Elle révèle une attitude équivoque au regard de l'intention de persister dans la formation à l'enseignement, dont les modalités de réalisation à distance apparaissent dissuasives. Elle révèle également une intention ferme de persister dans la profession enseignante, en relevant les défis que pose ce contexte de pandémie.

Mots-clés

Formation à l'enseignement, intention de persister, investissement professionnel, analyse de contenu, lexicométrie

Abstract

This article documents students' intentions to persist in a teacher training program and a career in preschool and elementary school education during the current pandemic. Interview data from a sample of 33 students were subjected to lexicometric content analysis. Results show ambiguous attitudes toward intention to persist in a teacher training program, notably due to the deterrent effect of remote learning modes. Results also reveal firm intentions to persist in the teaching profession while meeting the challenges posed by the pandemic.

\section{Keywords}

Teacher training, intention to persist, professional commitment, content analysis, lexicometry

\section{Introduction}

La pandémie actuelle a touché de plein fouet la profession enseignante et, indirectement, la formation à celle-ci. Les données disponibles sont certes parcellaires, mais, ici comme ailleurs, elles font néanmoins état de changements importants dans l'exercice du travail enseignant, comme la distanciation sociale, la transition vers des modalités d'enseignement à distance et le retour à des activités en présentiel sous le signe de mesures sanitaires rigoureuses (OCDE, 2020; Pramling Samuelsson, Wagner et Eriksen Ødegaard,2020). Au Québec comme ailleurs, la levée des activités d'enseignement en présentiel a obligé les enseignants à se familiariser avec les outils technologiques permettant de maintenir un rapport pédagogique avec leurs élèves (OCDE, 2020). Selon Goyette (2020), ces changements auraient affecté le bien-être et la motivation des enseignants, alors qu'ils " peuvent nourrir un sentiment d'incompétence numérique, ou un inconfort psychologique et émotionnel.»Dans les universités québécoises, cette mesure s'est traduite par la fin des activités d'enseignement en présentiel et des stages de formation. Néanmoins, plusieurs étudiants en éducation préscolaire et en enseignement primaire ont trouvé un emploi en milieu scolaire ${ }^{1}$ et ont été confrontés à des conditions de pratique inédites.

Ces conditions de travail et de formation à l'enseignement peuvent apparaître doublement problématiques aux yeux de ces étudiants. Non seulement celles-ci sont susceptibles d'affecter leur motivation à étudier, mais elles peuvent compromettre leur intention de poursuivre dans la profession enseignante. Ces conditions ne correspondent pas à leurs "savoirs préprofessionnels » en faveur d'une "pédagogie traditionnelle apprise sur les bancs de l'école comme élèves " (Tardif et Lessard, 2004, p. 381), laquelle s'est instaurée aux $\mathrm{XIX}^{\mathrm{e}}$ et $\mathrm{XX}^{\mathrm{e}}$ 
siècles dans la foulée de l'expansion des systèmes scolaires. À cet égard, un récent rapport de l'OCDE (2020) souligne que l'enseignement à distance pourrait faire encore partie des pratiques éducatives au cours des années à venir. Si un retour à la normale demeure envisageable, la perception de ces conditions peut compromettre momentanément le raisonnement circulaire présidant normalement à la reproduction des rôles scolaires. Cette situation soulève donc la question de l'intention de ces étudiants de poursuivre une carrière en enseignement. Ceux-ci «temporisent-ils » dans l'espoir d'un retour à la normale?

Le présent article a pour objectif de documenter l'intention de persister dans leur formation et la profession enseignante. Le construit d'intention de persister sera d'abord défini dans la prochaine partie dans la perspective de la théorie sociocognitive. Il permettra d'argumenter nos choix méthodologiques présentés dans un deuxième temps, en mettant en évidence les modalités de recueil et d'analyse des données, de nature lexicométrique. Les résultats des analyses permettront, dans un troisième temps, d'apporter des éléments de réponse, qui seront discutés par la suite.

\section{L'intention de persister dans la perspective de la théorie sociocognitive}

L'intention de demeurer dans la profession enseignante repose sur la motivation, les attitudes et les expériences des étudiants poursuivant un programme de formation à l'enseignement (Aksu, Demir, Daloglu, Yildririm et Kiraz, 2010; Heinz, 2015). C'est en fonction de ces facteurs que s'opérerait un mouvement constant de déconstruction et de reconstruction identitaire au cours de cette formation (Cochran-Smith, Villegas, Whalen Abrams, Chávez-Moreno, Mills et Stern, 2016). L'intention de persister correspond à la dimension conative de la disposition individuelle à agir : sans être l'action en elle-même, elle se trouve au plus près de celle-ci et de son contexte perçu par l'individu et affecte son désir de s'y engager. Nous avons recours à la théorie sociocognitive de Bandura (1986) pour l'analyser, alors qu'elle peut rendre compte de changements individuels au chapitre des dispositions cognitives, sociales et affectives dans la foulée de transformations contextuelles. La causalité établie par cette théorie repose sur l'interaction entre trois types de facteurs, respectivement de nature personnelle, environnementale et comportementale. Chacun de ceux-ci constitue autant de déterminants modérant l'effet des deux autres. Les facteurs individuels sont liés à la personne et renvoient à ses capacités, ses expériences antérieures et ses états émotionnels. Ils varieront en fonction des normes sociales prévalant dans un milieu spécifique et des comportements déployés par les individus pour agir.

Ces trois types de facteurs seront successivement abordés dans le but de situer les implications de la pandémie actuelle au regard de l'intention de persister. Il s'agit d'abord de prendre la mesure des changements comportementaux entraînés par celle-ci, suscitant diverses opinions individuelles. Nous aborderons ensuite la question de l'engagement en tant que disposition individuelle représentant la synthèse des facteurs individuels et sociaux, laquelle s'exprime à travers des attributions causales. Celleci permettra finalement de définir deux types d'intention.

\section{De nouveaux comportements en enseignement en contexte de pandémie}

Peu de recherches font systématiquement état des changements ayant affecté les différents volets de la tâche enseignante depuis les débuts de la pandémie actuelle. Certaines recherches prennent néanmoins la mesure de ceux-ci dans certains pays scandinaves, la Californie et la Thailande. Létude

2 - Formation et profession 28(4 hors-série), 2020 
comparative de Pramling Samuelsson, Wagner et Eriksen Ødegaard (2020) indique que certaines écoles préscolaires et primaires sont demeurées ouvertes en Suède, alors quelles ont généralement été fermées ailleurs. Les fermetures d'établissements ont d'ailleurs été massives à l'échelle internationale, affectant 1,3 milliard d'élèves de 182 pays en date du 3 mai 2020 (OCDE, 2020). Pour l'essentiel, il faut distinguer deux types de changements, correspondant aux deux phases de la crise actuelle, à savoir l'usage plus ou moins forcé des technologies de l'information à des fins d'enseignement dans un premier temps, et l'instauration de mesures sanitaires strictes dans le contexte de la reprise des activités en présentiel.

Le recours aux technologies de l'information s'est variablement imposé dans la foulée du confinement pour maintenir d'abord des canaux de communication avec les élèves et, graduellement pour plusieurs, pour élaborer des activités d'apprentissage en ligne, des enregistrements et des classes virtuelles à l'aide des plateformes informatiques disponibles (Pramling Samuelsson, Wagner et Eriksen Ødegaard, 2020). Ce changement a marqué un tournant important, considérant que seulement près de la moitié des enseignants recensés par l'OCDE (2020) ont affirmé avoir recours à ces technologies avant la crise. En outre, la réalisation effective d'enseignements en ligne a exigé une implication parentale accrue, qui était passablement limitée avant la crise (OCDE, 2020) et qui s'est avérée généralement variable au cours de celle-ci (Pramling Samuelsson, Wagner et Eriksen Ødegaard, 2020). L'adoption de ces outils technologiques a suscité des sentiments d'anxiété et d'incompétence (Pramling Samuelsson, Wagner et Eriksen Ødegaard, 2020). Nos étudiants ont été affectés par cette situation à la fois en tant que futurs enseignants et apprenants, la levée des cours universitaires en présentiel générant des sentiments d'incertitude et d'anxiété (Lee, 2020). Devant cette crise, Baloran (2020) souligne la nécessité de mieux préparer les étudiants en enseignement à faire usage des outils technologiques dans une perspective mixte pour soutenir les apprentissages en présentiel et à distance. Par ailleurs, la perte de la relation interpersonnelle en présentiel aurait durement été ressentie par les enseignants, considérant que « they view these relationships as the heart and soul of teaching » (Pramling Samuelsson, Wagner et Eriksen Ødegaard, 2020, s.p.). Certains ont exprimé des inquiétudes pour le bien-être et le développement de leurs élèves, privés de services scolaires adéquats et à risque d'être confrontés ultérieurement à d'importants défis académiques.

Par la suite, les comportements enseignants ont dû s'adapter aux mesures sanitaires présidant à la reprise des activités d'enseignement en présentiel, à savoir la distanciation sociale et le lavage fréquent des mains (Pramling Samuelsson, Wagner et Eriksen Ødegaard, 2020). Cette reprise a également été ponctuée par la réduction de la taille des groupes et du ratio entre l'enseignant et le nombre d'élèves. Ces mesures ont entrainé des comportements variablement appréciés par les enseignants. Les enseignants de la Norvège rendent compte de rapports plus chaleureux et personnalisés avec les enfants avec la diminution des ratios, permettant d'arrimer plus étroitement les situations d'apprentissage à leurs besoins et intérêts. La majorité constate cependant la lourdeur de la logistique entourant la mise en œuvre des nouvelles mesures d'hygiène et des protocoles sanitaires, impliquant de fréquents rappels aux élèves en matière de nettoyage des mains et prescrivant le nettoyage du matériel scolaire. Outre de la fatigue, ces comportements suscitent parfois la crainte chez les enseignants d'être harcelants pour les enfants, qui expriment parfois de l'exaspération.

Ce tour d'horizon des comportements conséquences de la pandémie actuelle permet donc de brosser un tableau contrasté. Si l'adoption plus ou moins forcée d'outils technologiques a d'abord eu des 
implications négativement perçues, elle a été suivie de comportements positivement interprétés dans le contexte de réduction de la taille des groupes.

\section{L'investissement professionnel en tant que disposition individuelle et rapport environnemental}

Définir l'engagement professionnel - qu'il serait plus approprié de qualifier d'investissement professionnel selon De Stercke (2014) - en tant que disposition individuelle est ambigu dans le contexte de cette recherche, car les écrits réfèrent variablement autant aux théories de la motivation au travail qu'à celles relatives au développement humain. À titre illustratif, Heinz (2015) s'appuie sur cette première perspective théorique dans sa recension systématique sur l'engagement professionnel des étudiants en formation initiale à l'enseignement. Comparativement, Klassen, Aldhafri, Mansfield, Purwanto, Siu, Wong et Woods-McConney (2012) ont recours à la théorie de l'autodétermination de Ryan et Deci (2000) dans le contexte de validation internationale d'un instrument de collecte. Aux fins de notre propos, nous retiendrons les attributs communs à ces deux approches théoriques, à savoir qu'il s'agit d'un état psychologique d'ordre motivationnel chez les individus, intervenant à la fois dans la relation d'attachement éprouvée chez ceux-ci à l'égard de leur profession et se caractérisant par la mobilisation de leurs forces laborieuses dans la réalisation des activités qu'elle implique (Meyer et Herscovitch,2001). Cette définition rejoint celle proposée par De Stercke (2014), à savoir l'attachement d'un enseignant à sa profession et aux finalités scolaires et sociétales associées à celle-ci, s'exprimant par sa participation volontaire et non contrainte aux tâches lui étant relatives, dont il est possible d'en apprécier la durée et l'intensité. Cette participation volontaire trouve son origine autant dans les dispositions individuelles que dans les rapports sociaux (environnement), qui déterminent la forme, l'orientation, l'intensité et la durée des activités laborieuses (Pinder, 1998, p. 11). La théorie de l'autodétermination précise que ces dispositions individuelles reposent sur trois types de besoins fondamentaux, à savoir l'autonomie, la compétence et l'appartenance. Ce serait en fonction de ces derniers et en référence à des normes ou des règles socialement partagées et variablement intériorisées que les individus attribuent un sens à leur action (Ryan et Deci, 2000).

Ces besoins fondamentaux peuvent rendre compte de motifs d'investissement dans des tâches notamment dans le contexte d'une formation à la profession enseignante, en plus d'être conformes au principe d'agentivité humaine sous-tendant la théorie sociocognitive (Bandura, 1986). Dans l'esprit de la théorie de l'autodétermination et de manière homologue à Heinz (2015), nous distinguerons trois types de motivation qui définiront autant de types d'attributions, à savoir intrinsèque, altruiste et extrinsèque ${ }^{3}$. Une motivation intrinsèque rend compte de comportements autodéterminés et réalisés par l'individu dans la perspective de préserver sa cohérence interne, au regard de ses besoins, de ses valeurs et de ses intérêts (Ryan et Deci, 2000). Elle renvoie aux facteurs proximaux de la relation pédagogique et du désir d'enseigner à des enfants, tels que le plaisir, l'enthousiasme, la créativité et le sentiment d'accomplissement. Tardif et Lessard (2004) ont rapporté à cet égard des propos exprimant ce « sentiment » d'unicité personnelle associé à l'exercice de l'enseignement dans le contexte spécifique de la relation pédagogique, lequel serait déjà présent «bien avant l'entrée en fonction, même avant la formation initiale» (p. 381). Ce sentiment puiserait ses racines dans des expériences antérieures positives à l'égard de l'école, ainsi que dans des expériences gratifiantes réalisées auprès des enfants. Les motivations altruistes, initialement documentées par Valentine (1934), relèvent de la perception de la valeur de la fonction sociale de l'enseignement, en tant que vecteur de progrès et d'intégration,

4 - Formation et profession 28(4 hors-série), 2020 
et de son rôle auprès des enfants et des adolescents. Pour leur part, Tardif et Lessard (2004) ont identifié une «mentalité de service » caractérisant le choix d'une carrière en enseignement. Ces deux types de motivation rejoignent en partie les trois cas de figure associés par Ryan et Deci (2000) à leur conception de la motivation intrinsèque, à savoir 1) la motivation intrinsèque à la stimulation découlant de sensations agréables associées à une activité; 2) la motivation intrinsèque à la connaissance marquée par le plaisir d'apprendre; et 3) la motivation intrinsèque à l'accomplissement caractérisée par le désir de relever un défi. Selon De Stercke (2014) s'appuyant sur Dupont, Carlier, Gérard et Delens (2009), la motivation intrinsèque de stimulation serait la plus importante dans le choix de carrière en enseignement.

La motivation sera considérée extrinsèque si les causes de l'action sont instrumentales, c'est-à-dire si les comportements sont d'abord déployés et orientés pour atteindre une récompense ou éviter une conséquence négativement perçue (Ryan et Deci, 2000). Une telle motivation serait dictée par le désir d'occuper un emploi perçu comme étant relativement stable et socialement reconnu, offrant une certaine flexibilité d'horaire et relativement bien rémunéré avec un fonds de pension, un régime d'assurances collectives et de généreuses vacances à la clé (Heinz, 2015). Règle générale, ce type de motivation serait moins fréquent chez les étudiants en enseignement, et tendrait même à être dévalorisé par ceux-ci (Lortie, 1975).

\section{L'intention de persister ou d'abandonner}

L'intention de persister ou d'abandonner est le prolongement conatif de l'investissement professionnel. En principe, des individus investis dans leur formation et à l'égard de la profession enseignante autant pour des motifs intrinsèques ou extrinsèques - exprimeront une intention de persister et non pas d'abandonner. La crise actuelle liée au coronavirus est une donnée contextuelle ayant entraîné des changements comportementaux importants susceptibles d'affecter cette intention de persister. En principe, les individus percevant favorablement ces changements exprimeront des attentes favorables à l'égard de leur profession et de leur formation, ainsi que des attributions expliquant un investissement (Simbula et Guglielmi, 2013). Dans le contexte du coronavirus, nous pouvons supposer que les étudiants ayant une intention de persister plus ferme seront disposés à en reconnaître les retombées positives, comme la réduction de la taille des groupes et les opportunités d'apprentissage, que ceux qui sont moins déterminés. Inversement, ceux dont la motivation aura été compromise exprimeront des opinions moins favorables et des attributions expliquant un désengagement. Ce cas de figure rejoindrait le troisième type de motivation identifié par Ryan et Deci (2000) est l'amotivation, caractérisée par une absence de motivation.

\section{Méthodologie}

Une analyse de contenu lexicométrique a été effectuée sur des corpus d'entrevues réalisées auprès d'un échantillon de convenance de 33 étudiants poursuivant ou ayant complété au terme de la dernière session universitaire un programme de formation à l'éducation préscolaire et à l'enseignement primaire. Ces étudiants proviennent de l'UQAM, l'UQTR et l'Université de Sherbrooke. Les entrevues ont été réalisées entre le 21 mai et le 9 juillet 2020 et la question suivante a été posée : «Indiquez si la pandémie actuelle de coronavirus est susceptible d'affecter votre intention de poursuivre une formation 
ou dans la profession enseignante. Précisez les raisons de ce choix.» Ce libellé demandait ainsi aux répondants de formuler une opinion sur leur formation ou la profession enseignante et une attribution causale permettant d'expliquer leur intention.

Les données ont été analysées par statistique textuelle, suivant notre objectif visant à déterminer des types de disposition individuelle, donnant lieu à des caractéristiques communes. Cette analyse permet de trouver des traits structuraux correspondant à des systèmes de valeurs au sein de discours commun (Lebart, Piron et Steiner, 2003). Concrètement, les données ont été traitées avec le logiciel DTM-Vic, ayant permis de segmenter les discours en mots individuels et de les soumettre à une analyse factorielle des correspondances. Cette opération statistique génère une représentation multidimensionnelle des mots soumis à l'analyse sous la forme d'un nuage de points (ou plan factoriel). Il faut comprendre que ce n'est pas la totalité du corpus qui est soumis à l'analyse, mais uniquement les mots dont la fréquence dans le texte est égale et supérieure à un seuil déterminé par le chercheur. Pour l'interprétation, le logiciel traverse ce dernier d'axes auxquels est attribuée une valeur propre, exprimant leur inertie respective à l'égard de la variance totale. L'interprétation est complétée par l'identification des mots présentant une inertie élevée, ainsi que l'examen de leur contexte dans le corpus.

\section{Résultats}

La figure 1 présente le plan factoriel généré par l'AFC et permet de constater la présence d'un discours commun. Le choix du seuil de fréquence a été déterminé en fonction des deux principales réponses attendues: oui ou non. Ces mots ont respectivement une fréquence de 14 et de 31 . En première approximation, nous pourrions supposer que les répondants semblent généralement peu affectés au chapitre de leur intention de poursuivre. Toutefois, une fréquence élevée ne permet pas d'avancer qu'elle n'est pas sans équivoque et qu’elle est uniformément répartie entre les répondants. Une analyse plus attentive est nécessaire des trois premiers axes, comptant respectivement pour 9,09\% (axe 1), 8,48 \% (axe 2) et 7,2\% (axe 3) de l'inertie totale du nuage de points.

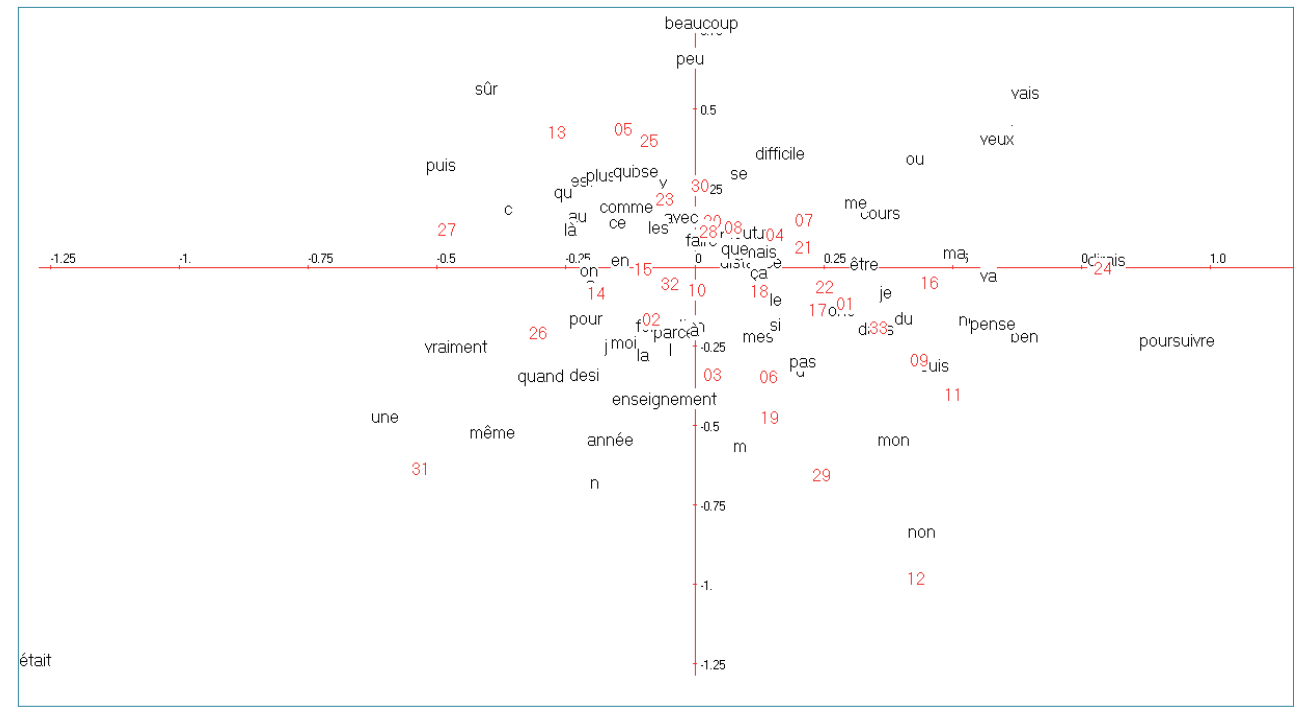

Figure 1

Plan factoriel des axes 1 et 2 - Intention de persister des étudiants en éducation préscolaire et en enseignement primaire

6 - Formation et profession 28(4 hors-série), 2020 


\section{Axe 1 : oui et non, ça dépend...}

Les mots oui (sous le mot veux) et non se côtoient en complémentarité et non en opposition dans la portion positive du premier axe, en position horizontale. Leur part respective de variance est d'ailleurs comparable, même si le non prédomine légèrement (oui : 1,$5 ;$ non : 1,8 ) en prenant en compte des mots relatifs à celui-ci (ne:5,9 et pas:1,5). Ils expriment essentiellement une attitude équivoque à l'égard de l'intention de poursuivre $(4,7):$ ben $(2,1), j e(7,5)$, dirais $(2,7)$, vais $(2,0)$, veux $(2,0)$, pense $(1,4)$, être $(1,4)$ et suis $(1,3)$. La poursuite renvoie à soi $(m a: 1,7$; mon : 1,1) et semble s'articuler particulièrement autour du mot va $(4,3)$. Un examen des concordances permet de situer cette inquiétude au chapitre de leur expérience de formation à l'enseignement en tant qu'étudiants, sans référence explicite ou directe à la profession enseignante. Cette préoccupation est partagée par les répondants dont la contribution est la plus significative $(24: 20,6 ; 16: 5,1 ; 33: 4,3 ; 7: 2,9 ; 21: 2,8 ; 11: 2,3 ; 09: 1,9 ; 01: 1,8 ; 07: 1,4$; $12: 1,3)$.

\section{Ben je vous dirais oui et non dans ce sens que je pense que je vais poursuivre quand même. Mais je me laisse la porte de ne pas poursuivre. Parce que moi ce qui me stresse beaucoup c'est la façon que le trimestre d'automne va se dérouler. (Sujet 24) \\ Celle-là est difficile! hi, hi, hi... ça dépend des jours! Je dirais dans l'ensemble non, parce que je compte vraiment finir. [...] c'est moins gratifiant de ne pas savoir si on va avoir des stages éventuellement ou pas. Comment ça va se passer au niveau des stages, parce que on a eu juste des bouts du stage du préscolaire, puis après ça, ça s'est coupé abruptement. Je dirais que oui, mais toute cette incertitude fait que je sois moins encouragée. (Sujet 07)}

La portion négative de cet axe rend plutôt compte des circonstances anxiogènes entourant à la fois l'exercice de la profession enseignante et de la formation à celle-ci au cours des prochains mois. Le mot était $(11,0)$ contribue le plus à sa variance, mais il est caractérisé par un effet de projection dans le plan factoriel (figure 1 ). Avec les mots $c(6,2)$, est $(3,5)$, une $(3,5)$, puis $(3,0)$, vraiment $(2,4)$ et même $(1,5)$, il décrit des sentiments variablement mitigés devant des situations de pratique et de formation :

Parce que depuis le 15 mars ce n'était pas clair les consignes du ministère, c'était flou, c'était vague, c'était... ils mont demandé de faire une classe en ligne, ce que j'ai fait. J'ai travaillé comme les collègues 10-12 heures par jour et je nétais pas rémunérée. J'ai fait ça parce que c'est vraiment une vocation pour moi, c'est essentiel pour moi d'être là pour des élèves, pour faire mon travail. Même si là ce n'était pas vraiment un travail, c'était bénévole, mais cétait important pour moi et je me sentais... utile on va dire. (Sujet 31)

Ca a été vraiment une adaptation dans les derniers mois, alors euh... quand il est venu le temps de finir la session, donc c'était vraiment stressant. (Sujet 27)

\section{Non, mais c'est sûr que le défi sera considérable...}

Le deuxième axe, vertical, exprime de manière sans équivoque une réponse négative à la question posée, alors que les mots non $(7,1), n(6,6)$ et pas $(3,3)$ indiquent que la pandémie actuelle ne semble pas avoir affecté l'intention des répondants de poursuivre leur formation ou dans la profession enseignante. Une intention qui, par ailleurs, est située dans le contexte d'un projet personnel, comme en témoignent 
les mots structurants $m(2,8)$ et mon $(2,3)$ exprimant le caractère déterminé de celle-ci. Elle traduit la contribution des sujets 31 (27,0), 12 (7,4), 29 (6,8), $19(6,3), 06(3,3), 03(2,7), 11(1,4)$ et $26(1,3)$ :

J'ai pris une classe à la fin de l'année pendant la pandémie pour les deux derniers mois. Je n'avais pas de problème à poursuivre, même dans les conditions actuelles. (Sujet 29)

Non! ça ne m’arrêtera pas! mais ça ne veut pas dire que ça ne suscite pas des craintes. (Sujet 06)

Une nuance doit cependant être apportée ici à l'égard des répondants 12 et 19, alors que le premier a déjà complété son baccalauréat et a entamé une maîtrise en éducation et que le second avait déjà décidé d'abandonner la profession avant la pandémie. Dans les deux cas, les mots structurants expriment davantage la fermeté de l'intention de poursuivre un projet professionnel, et moins la nature de celuici :

Dans la portion positive de ce deuxième axe, les mots est $(6,0)$, beaucoup $(4,4)$, peu $(3,6)$ et plus $(1,4)$ rendent compte des défis anticipés par les répondants, à la fois à l'égard de formation et de la profession enseignante. Ils sont particulièrement structurants au regard des contributions des sujets $05(14,7), 13$ $(6,4), 30(4,6), 25$ (3,5), $23(2,8), 28(1,8), 27(1,7)$ et $07(1,4)$. Les cours en ligne, dans le contexte d'un enseignement à distance, et la médiatisation des rapports plus largement semblent être les irritants les plus importants :

Je trouve ça difficile un peu ce qui est demandé en ce moment à tous les enseignants de la province. C'est-à-dire que... en faire encore beaucoup avec très peu et se débrouiller. Ce qui est selon moi un problème de société, de notre profession depuis longtemps. Puis là, c'est encore la même chose, mais avec plus. (Sujet 05)

Ca vient toucher beaucoup, beaucoup de sphères de toutes les vies de pas mal tout le monde (Sujet 30)

J'ai vraiment beaucoup de difficultés avec les médias, pas les médias sociaux, mais les plateformes comme ça, les plateformes internet. J'ai pratiqué beaucoup avec, je les ai utilisées, mais le contact bumain va beaucoup me manquer. (Sujet 23)

\section{Une formation démotivante pour une profession... motivante}

Le troisième axe, à la verticale du plan factoriel représenté par la figure 2 , exprime une opposition entre la formation et la profession. Si les attributions expriment une motivation intrinsèque des répondants dans la portion négative (inférieure), les mots va $(7,4)$, être $(5,5)$, pour $(4,4)$, pense $(3,7)$, puis $(2,3)$, cours $(1,9)$, ou $(1,6)$ et formation $(1,3)$ situés dans la portion positive (supérieure) rendent compte d'attributions fondées sur des anticipations au regard des modalités de formation qui seront mises en œuvre au cours de la prochaine session universitaire. 


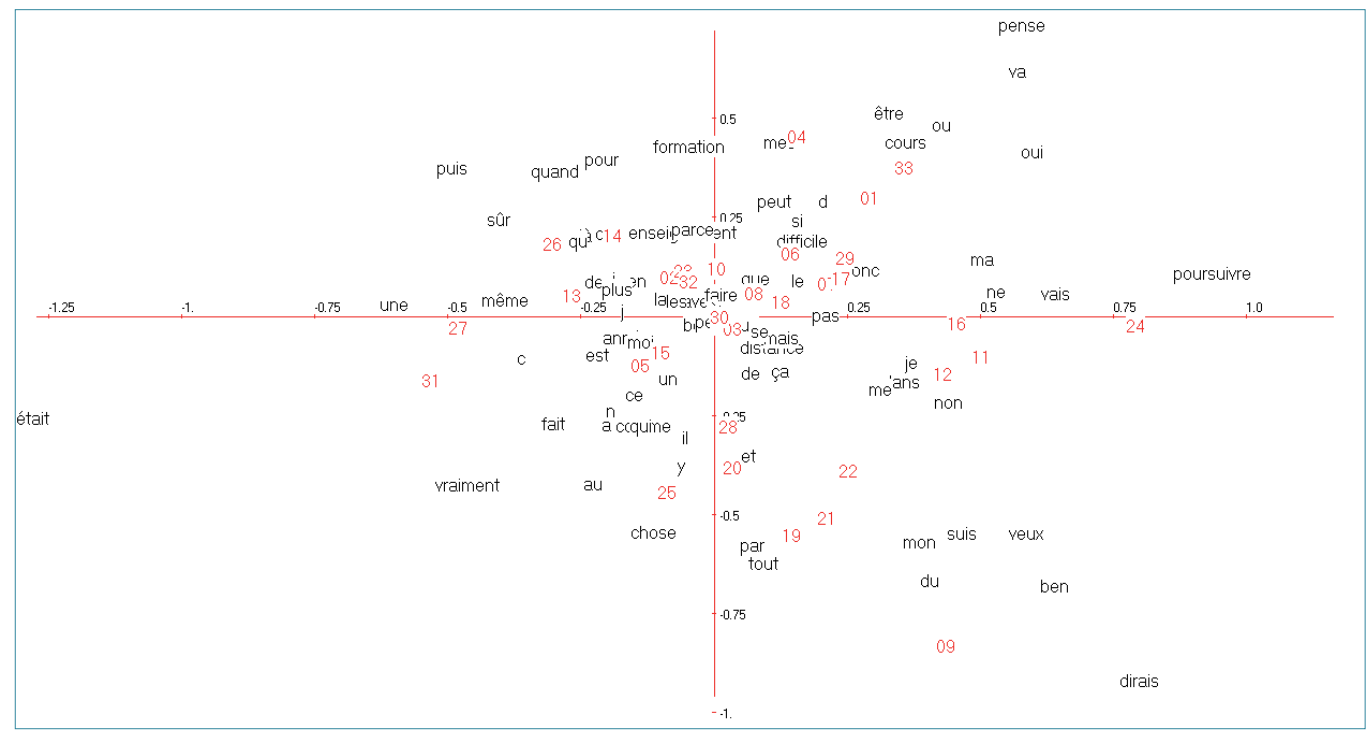

\section{Figure 2}

Plan factoriel des axes 1 et 3 - Intention de persister des étudiants en éducation préscolaire et en enseignement primaire

Particulièrement structurantes, les contributions des sujets $04(15,0), 33(7,8), 14(4,2), 26(2,2)$ et 17 $(1,2)$ mettent en relief trois causes susceptibles d'affecter variablement leur intention de poursuivre. Une dimension relationnelle est exprimée au regard du recours aux technologies de l'information en contexte de formation à l'enseignement et de stage. Celle-ci concerne autant le rapport pédagogique avec les élèves que celui avec les pairs en contexte de formation :

Puis pour les classes, c'est sûr que si on s'enligne pour quion donne des cours en ligne aux élèves pour le reste... Je ne pense pas que c'est ça que ça va être, mais... je ne sais pas. Ça, peut-être que j'irais changer, parce quion ne pourrait pas tant être en contact avec les enfants ou via internet là, ce n'est pas la même chose. J'aurais de bons questionnements à me faire, oui. (Sujet 04)

Juste le fait de faire des travaux d'équipe par teams ou des choses comme ça, ça va être un petit peu plus difficile. Moi, qui a besoin d'échanger avec mes amis quand on fait des travaux ou juste quand on révise. (Sujet 33)

Plus globalement, une interrogation persiste sur la qualité perçue des expériences de formation, notamment dans la foulée de celles vécues au cours de la session précédente. De ce point de vue, l'impression «de ne pas recevoir d'enseignement » et d'être laissé à soi-même semble être la source d'une « réflexion » pour certains, anticipant un allongement de la formation :

Euh... ç'a été une réflexion, à savoir si je continuais la formation. Parce que là, pour le moment ça été dit que ça allait être en ligne. Puis, de ce que j'ai expérimenté de la dernière session qui s'est terminée en ligne, j'ai trouvé ça vraiment dommage parce qu'au final on payait le même prix pour la session alors quion n'avait pas d'enseignement. (Sujet 26). 
C'est sûr que pour l'université, j'ai peur pour ma motivation, pour les cours en ligne là. Je ne sais pas, mais j'ai l'impression que justement, si je n'ai pas d'autres personnes que je vais pouvoir contacter puis vivre ça... bien mes amis là, avec qui je vais pouvoir vivre un petit peu... les cours là, ce n'est pas toujours évident. J'ai peur un petit peu de ce que ça va donner, puis que ça ne va pas justement me prendre plus que quatre ans pour faire mon cheminement. (Sujet 04)

La portion négative met en évidence l'intention de persister dans la profession enseignante sur la base d'une attribution interne aux répondants : quoi qu'il arrive, ceux-ci ont l'intention ferme de devenir enseignants. Les mots tout $(5,7)$, dirais $(4,7), m(4,3)$, ben $(3,1)$, mon $(3,1)$, suis $(2,4)$, veux $(2,2)$, chose $(1,9)$ et vraiment $(1,8)$ sont particulièrement structurants et mettent en évidence les contributions des répondants $28(6,1), 25(4,1)$ et $20(2,1)$, ainsi que celles plus spécifiques des répondants $21(22,1), 19$ $(10,5), 09(9,0)$ et $22(2,9)$. Globalement, elles expriment une intention de « relever le défi » :

Non, ça ne meempêchera pas de poursuivore. C'est sûr que ça me fait peur et c'est un autre défi de plus. Mais comme j'ai dit plus tôt je suis prête pour les défis et s'il y a un défi de plus, ben... on le prend. (Sujet 20)

Je veux pareil devenir enseignante. T'sé, je veux dire ce n'est pas quelque chose qui me décourage de le faire malgré toutes les mesures. C'est sûr que c'est un défi et que c'est plus difficile, mais c'est ça. (Sujet 25)

Ben je dirais que non que ça ne m’affectera pas malgré tout ce qui se passe. Je me suis questionné mais mon... mon objectif est clair. C'est vraiment d'aller chercher mon brevet et de continuer malgré la situation sociale et la situation économique de la profession. (Sujet 09)

Ca ne m'affecte pas parce que je suis encore convaincu que c'est la chose que je veux faire. (Sujet 21)

L'analyse des trois premiers axes a ainsi permet de mettre en lumière un portrait contrasté, alors que des réponses positives et négatives à la question se côtoient au premier axe, et que l'anticipation du défi à relever semble considérable au deuxième axe. Il n'est véritablement question de poursuivre ou d'abandonner qu'à partir du troisième axe.

\section{Discussion}

Cet article avait pour objectif de documenter l'intention de persister des étudiants en éducation préscolaire et en enseignement primaire. Une analyse de contenu lexicométrique des réponses formulées par un échantillon de 33 d'entre eux a permis de mettre en lumière quelques fondements d'opinions et d'attributions pour rendre compte de cette intention.

Le premier fondement semble relever de l'expérience de formation à l'enseignement, bien avant celui de la profession enseignante en elle-même. L'expression un tien vaut mieux que deux tu l'auras nous semble traduire le raisonnement argumentatif des répondants. Si certains répondants apparaissent intrinsèquement motivés, comme constaté lors de l'analyse du troisième axe, c'est d'abord cette expérience qui semble être déterminante au regard de l'intention de poursuivre. Le fait que l'expérience plus ou moins désagréable de la session d'hiver soit encore fraîche dans les esprits et quelle donne un avant-goût de celle d'automne pourrait expliquer ce résultat : l'intention devient équivoque devant des 
stresseurs environnementaux. En contexte de formation, ceux-ci apparaissent proximaux et mettent en évidence le poids de la dimension relationnelle qui, autant en contexte de formation que de profession, est de la même venue. Par ailleurs, nous remarquons que la nature des changements comportementaux causés par la pandémie est peu définie dans les discours analysés.

L'intention de poursuivre s'articule à un deuxième fondement de nature motivationnelle, constaté aux deuxième et troisième axes, à savoir celui de relever un défi. Il rejoint le troisième type de motivation intrinsèque identifié par Ryan et Deci (2000) caractérisé par le désir de relever un défi. Même si celuici suscite des craintes, il est significativement associé à une réponse négative à la question posée. D'un point de vue sociocognitif, le fait qu'il y ait défi ne semble pas perçu comme un problème, mais une source de motivation et d'attribution pour rendre compte d'un attachement personnel à la profession. Cependant, nos résultats ne permettent pas de considérer que cette relation d'attachement soit fondée sur les finalités scolaires et sociétales relatives à la profession. En principe, cette relation entretiendrait une relation dynamique avec la qualité perçue des expériences professionnelles, la satisfaction et l'attachement à la profession (Li, Wang, Gao et You, 2015). Elle affecterait la nature des objectifs que les individus se donnent d'atteindre, ainsi que l'intensité et la persistance de leurs efforts (Klassen et Chiu, 2011). Considérant que les récentes expériences de formation semblent être négativement perçues, il se pourrait que ce désir de relever le défi soit un fondement fragile. En un sens, exprimer un tel désir pourrait traduire une manière de «temporiser » ou de " gagner du temps ». Certains propos des répondants sur l'impact de l'enseignement à distance à l'égard de leur motivation laissent présager un désengagement si la situation devait se prolonger. Selon De Stercke (2014), s'appuyant sur Rots, Aelterman, Vlerick et Vermeulen (2007), les attentes à l'égard de la formation initiale contribueraient à renforcer l'investissement professionnel, lequel serait influencé par les caractéristiques de la formation à l'enseignement et les premières expériences d'enseignement.

\section{Conclusion}

Cette recherche avait pour objectif de documenter l'intention de persister dans la formation et la profession enseignante d'étudiants en éducation préscolaire et enseignement primaire. Une analyse lexicométrique des données a révélé des opinions équivoques au regard des changements ayant affecté les modalités de formation, mais également une intention de persister dans la profession pour relever les défis impliqués par la pandémie actuelle. Il apparaît donc raisonnable de supposer que les conséquences sur la poursuite des études et l'insertion professionnelle seront relativement modérées. Toutefois, certaines données indiquent que cette intention pourrait être plus fragile pour les étudiants dont la motivation apparaît plus faible. L'échantillon permettant d'avancer cette hypothèse est cependant petit. Une analyse plus poussée, appuyée sur des données plus abondantes et en documentant d'autres variables motivationnelles, comme le sentiment d'efficacité personnelle et le stress, autoriserait une interprétation plus nuancée. Pour l'instant, cette analyse invite à réfléchir à nos dispositifs de formation pour la session qui s'annonce. 


\section{Notes}

1 Au terme d'une réflexion étymologique et d'une recension des écrits sur la profession enseignante, cet auteur invite soit à utiliser l'expression investissement professionnel, soit à conserver celle anglophone de teaching commitment, pour conserver sa signification originale reconnaissant le caractère volontaire de ce construit. La relation d'attachement et la participation effectivement ne sauraient être établies sous la contrainte dans le contexte de l'investissement professionnel en enseignement.

2 La théorie de l'autodétermination de Ryan et Deci (2000) est d'une facture plus complexe que l'usage que nous en faisons. Celle-ci décrit plutôt un continuum que des catégories distinctes de motivation, dont les pôles sont la motivation intrinsèque et l'amotivation, le comportement étant complètement autodéterminé dans le premier cas, contrairement au second. Il se trouve différents types de régulation du comportement entre ces deux pôles, mais ceux-ci n’ont pas présidé à la cueillette et l'analyse des résultats.

3 Selon Christine Hamel de l'Université Laval, c'est environ 50 \% des 158 finissants de l'Université Laval qui auraient trouvé une classe pour le 11 mai 2020, https://nouvelles.ulaval.ca/societe/devenir-prof-durant-une-pandemie-8f21ef262018c2e2b57789e6f21ed820.

\section{Références}

Aksu, M., Demir, C.E., Daloglu, A., Yildririm, S. et Kiraz, E. (2010). Who are the future teachers in Turkey? Characteristics of entering student teachers. International journal of educational development, 30, 91-101. https:// doi:10.1016/j.ijedudev.2009.06.005

Baloran, E.T. (2020). Knowledge, attitudes, anxiety, and coping strategies of students during COVID-19 pandemic. Journal of loss and trauma. https://doi.org/10.1080/15325024.2020.1769300

Bandura, A. (1986). Social foundations of thought and action: a social cognitive theory. Upper Saddle River, NJ: Prentice Hall.

Cochran-Smith, M., Villegas, A.M., Whalen Abrams, L., Chávez-Moreno, L.C., Mills, T. et Stern, R. (2016). Research on teacher preparation: charting the landscape of a sprawling field. Dans D.H. Gitomer et C.A. Bell (dir.), Handbook of research on teaching (p.439-547). American educational research association.

De Stercke, J. (2014). Persévérance et abandon des enseignants débutants : la relève issue des Hautes Écoles (thèse de doctorat non publiée). Université de Mons-Hainaut, Mons, Belgique.

Dupont, J.P., Carlier, G., Gérard, P. et Delens, C. (2009). Déterminants et effets de la motivation des élèves en éducation physique : revue de littérature. Les cabiers de la recherche en éducation et formation, 73, 1-32.

Goyette, N. (2020, 8 avril). Qu'en est-il du bien-être des enseignants durant cette période de pandémie? Le Droit. https:// www.ledroit.com/opinions/votre-opinion/quen-est-il-du-bien-etre-des-enseignants-durant-cette-periode-depandemie-05c2d83ff9e615333116ab1cbbd88e16

Heinz, M. (2015). Why choose teaching? An international review of empirical studies exploring student teachers' career motivations and levels of commitment to teaching. Educational research and evaluation, 21(3), 258-297. https://doi. org/10.1080/13803611.2015.1018278

Klassen, R.M., Aldhafri, S., Mansfield, C.F., Purwanto, E., Siu, A.F.Y., Wong, M.W. et Woods-McConney, A. (2012). Measurement, statistics, and research design. Teachers' engagement at work: an international validation study. The journal of experimental education, 80(4), 317-337. https://doi.org/10.1080/00220973.2012.678409

Klassen, R.M. et Chiu, M.M. (2011). The Occupational commitment and intention to quit of practicing and pre-service teachers: Influence of self-efficacy, job stress, and teaching context. Contemporary Educational Psychology, 36(2), 114-129. https://doi/10.1016/i.cedpsych.2011.01.002

Li, M., Wang, Z., Gao, J. et You, X. (2015). Proactive personality and job satisfaction: The Mediating Effects of self-efficacy and work engagement in teachers. Current Psychology, 36(1), 48-55.

Lebart, L., Piron, M. et Steiner, J.-F. (2003). Sémiométrie. Dunod. 
Lee, J. (2020). Mental health effects of school closures during COVID-19. The Lancet, 4(6), 421. https://doi.org/10.1016/S2352-4642(20)30109-7

Lortie, D.C. (1975/2002). Schoolteacher: a sociological study (2 ${ }^{\mathrm{e}}$ éd.). University of Chicago Press.

Meyer, J. P., et Herscovitch, L. (2001). Commitment in the workplace: Toward a general model. Human Resource Management Review, 11, 299-326.

Pinder, C.C. (1998). Work motivation in organizational behavior. Prentice Hall.

Pramling Samuelsson, I., Wagner, J.T. et Eriksen Ødegaard, E. (2020). The coronavirus pandemic and lessons learned in preschools in Norway, Sweden and the United States: OMEP Policy forum. International journal of early childhood. https://doi.org/10.1007/s13158-020-00267-3

OCDE (2020). How prepared are teachers and schools to face the changes to learning caused by the coronavirus pandemic? Teaching in focus, 32. https://doi.org/10.1787/2fe27ad7-en

Rots, I., Aelterman, A., Vlerick, P. et Vermeulen, K. (2007). Teacher education, graduates' teaching commitment and entrance into the teaching profession. Teaching and teacher education, 23, 543-556.

Ryan, R. M. et Deci, E. L. (2000). Self-determination theory and the facilitation of intrinsic motivation, social development, and well-being. American Psychologist, 55(1), 68-78. https://doi.org/10.1037/0003-066X.55.1.68

Simbula, S. et Guglielmi, D. (2013). I am engaged, I feel good, and I go the extra-mile: Reciprocal relationships between work engagement and consequences. Journal of Work and Organizational Psychology, 29(3), 117-125.

Tardif, M. et Lessard, C. (2004). Le travail enseignant au quotidien. Contributions à l'étude du travail dans les métiers et les professions d'interactions humaines. Presses de l'Université Laval.

Valentine, C.W. (1934). An enquiry as to reasons for the choice of the teaching profession by university students. British Journal of Educational Psychology, 4, 237-259.

\section{Pour citer cet article}

Moreau, D., Larose, F. et Smith, J. (2020) L'intention de persister d'étudiants québécois en éducation préscolaire et en enseignement primaire en contexte de pandémie. Formation et profession, 28(4 hors-série), 1-13.

http://dx.doi.org/10.18162/fp.2020.687 